\title{
Applying a Hybrid Approach Based on Analytic Hierarchy Process and Artificial Neural Network to Upholstered Furniture Design
}

\author{
Ming Chen, Jianhua Lyu * and Jiabi Chen \\ College of Forestry, Sichuan Agricultural University, Chengdu 611130, China \\ *Corresponding Author: Jianhua Lyu, ljh@sicau.edu.cn
}

\begin{abstract}
Upholstered furniture evaluation is a complex and multi solution problem. Multi objective evaluation research can effectively improve the design efficiency and quality of upholstered furniture. The evaluation of each individual design candidate in terms of its ability to meet the demands of all factors should be taken into consideration and is a crucial step within the conceptual and product design stage. The evaluation indexes are established according to the principle of upholstered furniture design. Consequently, this paper proposes a method which enables upholstered furniture product evaluation by means of analytic hierarchy process $(A H P)$ and back propagation neural network (BP neural network) method of artificial neural network. The upholstered furniture indexes and the indexes weights are given by AHP, the BP neural network model of upholstered furniture are established by using the training sample data obtained from weight values of AHP. 30 upholstered furniture design proposals are chosen as the subject of this research, and 90 rating data are obtained by expert investigation method. 60 of them are used as training sample data and 30 of them are used as test sample data in BP neural network by MATLAB software program. Eventually, BP artificial neural network model for upholstered furniture evaluation was established by sample data training and testing in neural network with MATLAB, which can be used for simulation of upholstered furniture design quality and decision-making.
\end{abstract}

Keywords: Upholstered Furniture Design, Evaluation, Analytic Hierarchy Process, Artificial Neural Network, MATLAB Program

\section{Introduction}

Upholstered furniture is widely used in work and living environment. Upholstered furniture refers to a furniture category which uses wood or metal material as framework, and use spring, elastic bandage and foam as load-bearing material, surface covering with leather, fabrics and chemical fiber. There are many factors affecting the quality of upholstered furniture design quality, which makes quality evaluation and product decision difficult and unreasonable. Product evaluation or decision for designers and product preference and choice for costumers, in a way, it is a matter of one thing with two aspects, and both are indispensable. However, the way that consumers look at product is usually different from the way that designers look at product elements or characteristics [1]. Consumer-oriented technologies have been developed to help designers design a desired product to meet consumers' need of a product from a design perspective [2,3]. Particularly, Kansei Engineering has been applied successfully in the design-related fields to explore the relationship between the feeling of the consumers and the design elements of the product with translating consumer's feelings into design elements [4-8].

Recently, genetic algorithms have been applied in design-related fields. For example, an automatic design system for rapidly obtaining a product form and its corresponding 
image by using fuzzy neural networks and genetic algorithms was developed [9]; an approach using genetic algorithms for preliminary cam design and for subsequent shape optimization was developed [10]; genetic algorithms to fitting curves used in automotive body design was applied [11]; an approach by applying genetic algorithms and Kansei Engineering Type II (AHP and Quantification Theory Type I) with MATLAB program was developed [12].

In this paper, a hybrid approach was presented for answering specific research questions in upholstered furniture product design with respect to product design elements and customer needs, using analytic hierarchy process (AHP) and BP neural network method of artificial neural network techniques. These research questions include (a) how the product elements affect a particular image and quality of the upholstered furniture, (b) how many evaluation indexes can be used for upholstered furniture design, and (c) what technique should be used to help product designers or manufacturers determine or choose the best project alternative for given upholstered furniture. To illustrate how the approach can answer these questions, we conduct an experimental study on sofas, one category of upholstered furniture with MATLAB software.

\section{Mathematical Model of Comprehensive Evaluation in Upholstered Furniture Design}

Upholstered furniture design comprehensive evaluation is to make judge on overall quality of upholstered furniture design with related design, material, engineering, marketing theory and technology, with the purpose of improving the quality and level of upholstered furniture design. Design is a dynamic process, and there are many factors affecting the quality of design with different extent of influence on design project, which leads to the complicated nonlinear relation between the input and output of upholstered furniture design comprehensive evaluation, and it is difficult to establish a reasonable, precise mathematical analytical formula. Then mathematical model of upholstered furniture design comprehensive evaluation are as follows:

$$
y=f\left(x_{1}, x_{2}, \cdots, x_{n}\right)
$$

Here, $x$ refers to the evaluation index, $y$ refers to the evaluation function and result. The modeling of upholstered furniture design comprehensive evaluation is to find the most adaptive $f($ ), actually, which is able to depict the complex nonlinear relationship between the input and output of upholstered furniture design comprehensive evaluation system. The BP neural network has been used on function approximation in this paper, for the BP neural network has good nonlinear learning ability.

\section{Upholstered Furniture Design Comprehensive Evaluation Based on AHP-BP Neural Network}

\subsection{Evaluation Index System}

The first step to build upholstered furniture design comprehensive evaluation model is to establish the corresponding evaluation index system. Whether the evaluation index is scientific or not is directly related to scientific and practical properties of evaluation model. Upholstered furniture design comprehensive evaluation indexes are affected by many factors, such as style, form, function, color, material, surface decoration, structure, and economic value, etc.

According to analysis of upholstered furniture design elements by Delphi Method and consumer's needs by Questionnaire Method that has been used in this paper, as well as relevant references, upholstered furniture design comprehensive evaluation indexes are established by AHP method as Table1 shows. 
Table 1. Upholstered Furniture Design Evaluation Index

\begin{tabular}{|c|c|c|}
\hline & Second-class index & Third-class index \\
\hline & \multirow{2}{*}{ Style(B1) } & Suitable for consumer age(D1) \\
\hline & & Embodying social status and taste of the consumer(D2) \\
\hline & \multirow{3}{*}{ Morphology(B2) } & Morphology in conformity with function(E1) \\
\hline & & Beautiful line shape and new form(E2) \\
\hline & & $\begin{array}{l}\text { Symmetrical balance in morphology and harmonious } \\
\text { proportion in size(E3) }\end{array}$ \\
\hline & \multirow{3}{*}{ Color(B3) } & Popular colors(F1) \\
\hline & & Compatible with the environment and people(F2) \\
\hline & & Harmonious and vivid in color(F3) \\
\hline & \multirow{3}{*}{$\begin{array}{c}\text { Surface } \\
\text { decoration }(B 4)\end{array}$} & Harmony in the whole and the part(G1) \\
\hline & & Exquisite and artistic quality(G2) \\
\hline & & Accordance with the safety requirements of the user(G3) \\
\hline & \multirow{2}{*}{ Function(B5) } & Operated easily and flexibly and detachable(H1) \\
\hline & & Conforms to the principle of Ergonomics(H2) \\
\hline & \multirow{2}{*}{ Structure(B6) } & Adaptive capacity to materials(I1) \\
\hline & & Proper structure and structural stability(I2) \\
\hline & \multirow{8}{*}{ Material(B7) } & Fire resistance of surface and filling material(J1) \\
\hline & & Ignition resistance of surface and filling material(J2) \\
\hline & & $\begin{array}{c}\text { Abrasive resistance and color fastness of surface material(not } \\
\text { less than level four)(J3) }\end{array}$ \\
\hline & & Contact comfort and breathability(J4) \\
\hline & & Easy to clean for surface material(J5) \\
\hline & & $\begin{array}{c}\text { Environmental protection in filling material and safety for } \\
\text { human health(J6) }\end{array}$ \\
\hline & & Compressibility and resilience behavior in filling material(J7) \\
\hline & & $\begin{array}{l}\text { Application of Hi-tech material and technology, such as, } \\
\text { massage technology, mute technology, control technology, } \\
\text { antimicrobial properties(J8) }\end{array}$ \\
\hline & \multirow{3}{*}{ Economic value $(\mathbf{B 8})$} & Production cost $(\mathrm{K} 1)$ \\
\hline & & Difficult to imitate with innovation(K2) \\
\hline & & Obvious selling points with trailblazing(k3) \\
\hline
\end{tabular}

\subsection{Evaluation Index Determination Based on AHP}

The Analytic Hierarchy Process (AHP) enables decision-makers to structure a complex problem in the form of a simple hierarchy and to evaluate a large number of quantitative and qualitative factors in a systematic manner under conflicting multiple criteria. In AHP, an optimal alternative is obtained using the following three stages: decomposition, comparative judgments, and synthesis of priorities [13]. First, the complex and multicriteria problem is broken down into a number of small constituent elements, which are structured in a hierarchical form. In this hierarchy, the overall goal (usually the selection of the best alternative) is situated at the highest level, elements with similar features (usually evaluation criteria) are grouped at the same middle level, and decision variables (usually candidate alternatives) are situated at the lowest level. Then, a series of pairwise comparisons is made among the elements at the same level using the ratio scales 1, 3, 5, 7, and 9, which are suggested [13,14], and judgment matrices are formulated for all evaluation criteria. Finally, the relative weights of the criteria are estimated by calculating the eigenvalues for the judgment matrices with these relative weights aggregated and synthesized for the final measurement of given decision alternatives. 


\subsubsection{Determine the Weights of Each Criterion}

AHP provides a mathematically structured means for comparing the relative attributes and importance between two arbitrarily selected items. In this paper, this pairwise comparison method is utilized to quantify the linguistic qualitative expressions for each objective. Assume that $\mathrm{n}$ items, $s_{1} ; s_{2} \ldots s_{n}$, are supposed to compare the relative importance ratio of two selected items. If $\mathrm{n}$ importance grades, $a_{1} ; a_{2} ; \ldots ; ; a_{n}$, are associated with $\mathrm{n}$ items, the importance ratio, $a_{i j}$, denotes the $i$ th importance grade divided by the $j$ th importance grade and is formulated as:

$$
a_{i j}=\frac{a_{i}}{a_{j}}
$$

The importance ratios, $a_{i j}$, are generally defined on a scale from 1 to 9 . The comparison value 1 denotes that the $i$ th item is as important as the $j$ th item, while 9 denotes that the $i$ th item is extremely more important than the $j$ th item, as shown in Table 2.

\section{Table 2. Linguistic Definition for Importance Ratios of Two Selected Items}

\begin{tabular}{|c|c|}
\hline $\boldsymbol{a}_{i j}$ & $\begin{array}{c}\text { Linguistic definition for comparison of the } \boldsymbol{i} \text { th and the } \boldsymbol{j} \text { th } \\
\text { items }\end{array}$ \\
\hline $\mathbf{1}$ & The $i$ th item is equally important as the $j$ th item \\
\hline $\mathbf{3}$ & The $i$ th item is slightly more important than the $j$ th item \\
\hline $\mathbf{5}$ & The $i$ th item is more important than the $j$ th item \\
\hline $\mathbf{6}$ & The $i$ th item is strongly more important than the $j$ th item \\
\hline $\mathbf{9}$ & The $i$ th item is extremely more important than the $j$ th item \\
\hline $\mathbf{2 , 4 , 6 , 8}$ & The intermediate values between two adjacent judgments \\
\hline $\mathbf{1} / \boldsymbol{a}_{\boldsymbol{i} \boldsymbol{j}}=\boldsymbol{a}_{\boldsymbol{j} i}$ & The inverse comparison between the $i$ th and the $j$ th items \\
\hline
\end{tabular}

\subsubsection{Weighing Values for Evaluation Index and Consistency Inspection}

Weighing values for evaluation index and consistency inspection: Using the eigenvalue solution in AHP, the weight set $W=\{W 1, W 2, \ldots, W m\}$, is viewed as an eigenvector for eigenvalue $\lambda$, described as:

$$
A W=\lambda W
$$

The equation above can be simplified as:

$$
(A-\lambda I) W=0
$$

where I is an $n \times n$ identity matrix. From this equation, $\mathrm{W}$ is not a zero vector, and there are $\mathrm{n}$ solutions $(\lambda 1, \lambda 2, \ldots, \lambda \mathrm{m})$ for eigenvalue $\lambda$. Generally, the maximum eigenvaue, $\lambda_{\max }$, is taken, and its corresponding eigenvector $W$ represents the importance weights for $n$ items. In order to avoid inconsistency in the data, the importance comparison matrix $\mathrm{A}$ is used. The consistency ratio (CR) is used to examine the consistency of a matrix. CR is defined as:

$$
C R=C I / R I
$$

Where CI represents a consistency index and RI represents a random index. The CI values are calculated using:

$$
C I=\left(\lambda_{\max }-n\right) / n-1
$$


Where $n$ is the order of matrix $\mathrm{A}$. If the $\mathrm{CR}$ value for matrix $\mathrm{A}$ is less than 0.1 , the matrix is considered to have good consistency for calculating the weights of the items.

\subsubsection{Important Index Selection}

According to the ordering on weight influence of each evaluation index to final upholstered furniture design comprehensive evaluation result, important indexes are screened out as the BP neural network input, and the input information-dimensions of neural network are reduced and the learning speed of neural network are improved, which develop upholstered furniture design comprehensive evaluation system accuracy and efficiency.

\subsection{BP Neural Network Model}

The BP neural network is a kind of back propagation neural network, it consists of input layer, hidden layer and output layer, which has been become one of the most widely used artificial neural network, and the BP neural network structure is shown in Figure 1.

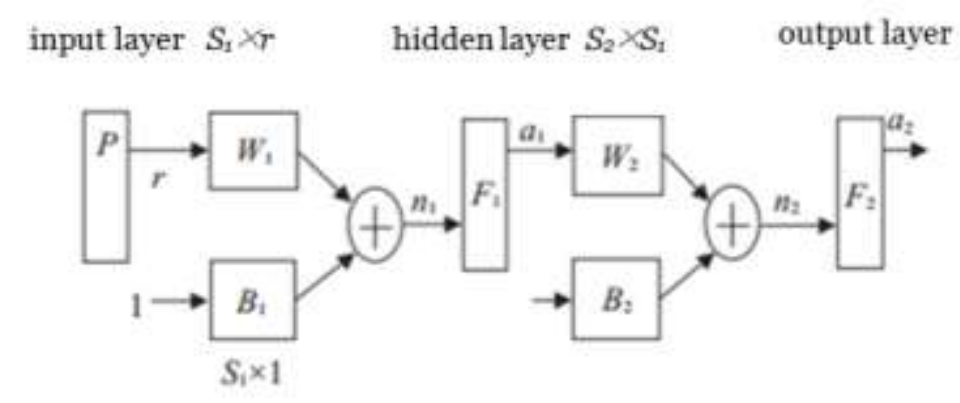

Figure 1. Structure of BP Neural Network

Output of input layer in BP neural network, described as:

$$
O_{j}^{(1)}=x(j)
$$

Input and output of hidden layer in BP neural network, described as:

$$
\left\{\begin{array}{l}
n e t_{i}^{(2)}(k)=\sum_{j=0}^{M} w_{i j}^{(2)} O_{j}^{(1)} \\
O_{i}^{(2)}(k)=f\left(n e t_{i}^{(2)}(k)\right)
\end{array}\right.
$$

Activation function of neuron in hidden layer approached by symmetric positive and negative Sigmoid function, described as:

$$
f(x)=\tanh (x)=\frac{e^{x}-e^{-x}}{e^{x}+e^{-x}}
$$

Input and output of output layer in BP neural network, described as:

$$
\left\{\begin{array}{l}
n e t_{1}^{(3)}(k)=\sum_{i=1}^{Q} w_{l i}^{(3)} O_{i}^{(2)}(k) \\
O_{l}^{(3)}(k)=g\left(n e t_{l}^{(3)}(k)\right)
\end{array}\right.
$$


Activation function of neuron in output layer approached by non-negative Sigmoid function, described as:

$$
g(x)=\frac{e^{x}}{e^{x}+e^{-x}}
$$

\subsection{Upholstered Furniture Design Comprehensive Evaluation Procedure Based on AHP-BP Neural Network}

Step 1 On the basis of analysis of upholstered furniture design elements by Delphi Method and consumer's needs by Questionnaire Method that has been used in this paper, as well as relevant references, evaluation index system is established by AHP.

Step 2 AHP is used to calculate comprehensive weight of upholstered furniture design comprehensive evaluation index, and sorting is conducted according to the importance of the index weights.

Step 3 Based on the evaluation index weight, and using the screening method, evaluation indexes that has major influence on the result of evaluation are chosen.

Step 4 According to the evaluation index screened by AHP, the number of neurons in BP neural network output layer is determined, and the gradation of upholstered furniture design comprehensive evaluation is taken as the output of model. The number of neurons in the hidden layer can be determined by the method of gradual growth, thus determining topology of BP neural network model.

Step 5 Normalization processing of index is performed to eliminate the adverse effects dimensional differences of index.

Step 6 BP neural network parameters are initialized, and adequate samples are selected, and then training and learning is conducted by using BP neural network, in order to establish the evaluation model.

Step 7. The established evaluation model is employed to evaluate upholstered furniture design project quality, with evaluation results output, and its performances are analyzed. AHP-BP neural network upholstered furniture design comprehensive evaluation process is shown in Figure 2. 


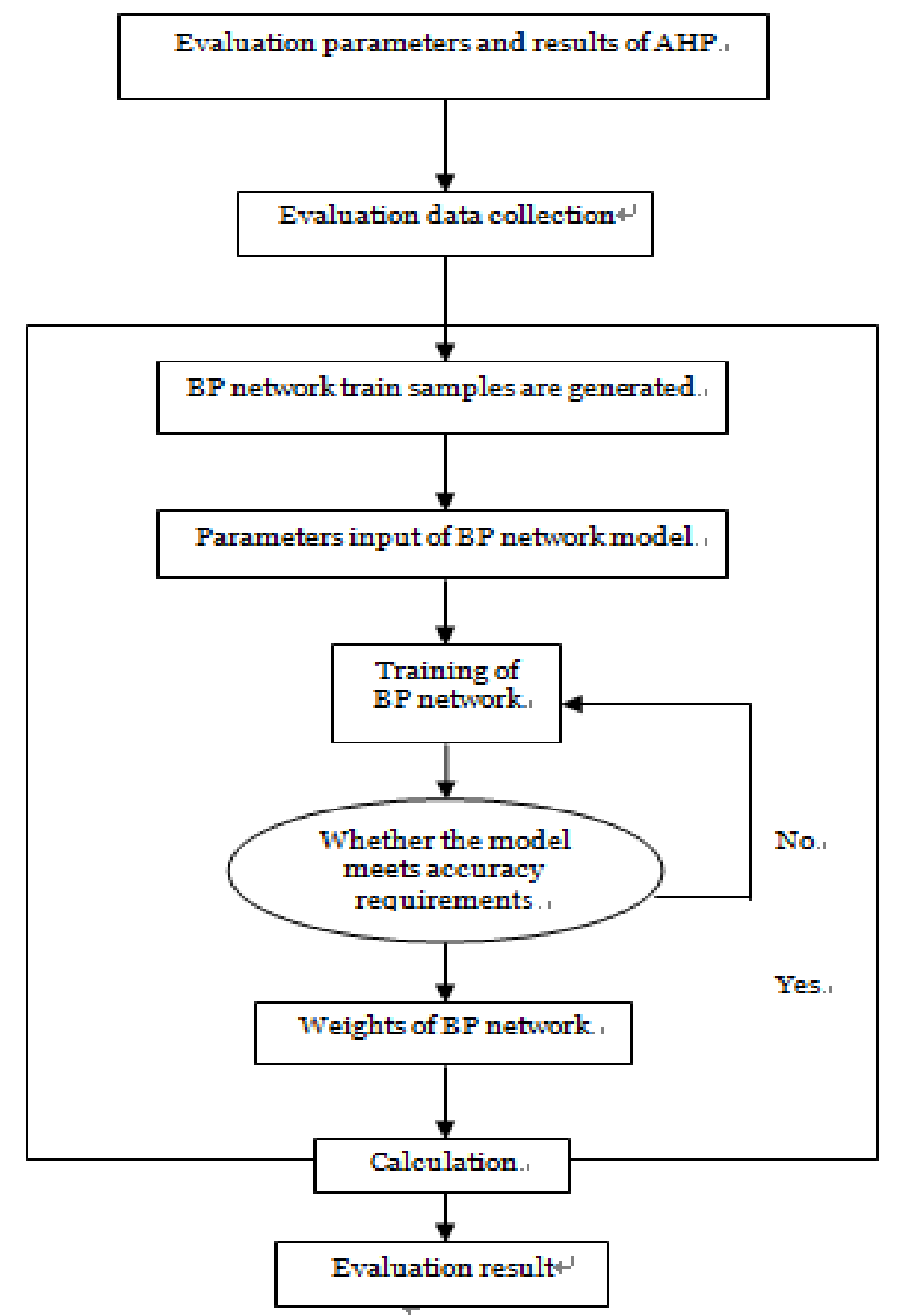

Figure 2. AHP-BP Neural Network Upholstered Furniture Design Comprehensive Evaluation Process

\section{Application of AHP-BP Neural Network in Upholstered Furniture Design Comprehensive Evaluation}

\subsection{Data Sources and Weights}

To test the performance of upholstered furniture design comprehensive evaluation based on AHP-BP neural network algorithm, simulation experiment is made in the upholstered furniture design comprehensive evaluation data which are collected by individual interviewing method. All the 40 participants are from Sichuan Province of China, their occupations are as follows: 15 furniture designer, $37.5 \%$ of participants, 18 design university teachers, $45 \%$ of participants and 7 design managers, $17.5 \%$ of participants, respectively.

Comprehensive weights are calculated by AHP. The comprehensive weights of indexes are shown in Table 3. The results in Table 4 shows that the consistencies of the matrix are less than 0.1 , the matrix have good consistency for calculating the weights of the items. Then, additional 30 design project alternatives are selected as evaluation projects, which 
are divided into 6 groups, and 3 upholstered furniture experts are assigned as evaluation experts per group. With a total of 90 data collected, the data are divided into two parts, in which 60 data are randomly selected as the training sample set, and the remaining 30 data are taken as the testing sample set. Each data includes a total of 26 evaluation indexes, and the upholstered furniture design comprehensive evaluation results can be divided into five different grades: excellent, good, middle, poor, worse, represented by 1,2,3,4 and 5 respectively. Part of the data is shown in Table 5.

Table 3.The Comprehensive Weight of Index

\begin{tabular}{|c|c|c|c|}
\hline \multicolumn{2}{|c|}{ B layer } & \multicolumn{2}{|c|}{ C layer } \\
\hline \multirow{2}{*}{ Style(B1) } & \multirow{2}{*}{0.0616} & D1 & 0.04928 \\
\hline & & D2 & 0.01232 \\
\hline \multirow{3}{*}{$\begin{array}{c}\text { Morphology } \\
\text { (B2) }\end{array}$} & \multirow{3}{*}{0.2773} & E1 & 0.05399 \\
\hline & & E2 & 0.02443 \\
\hline & & E3 & 0.19887 \\
\hline \multirow{3}{*}{$\begin{array}{l}\text { Color } \\
\text { (B3) }\end{array}$} & \multirow{3}{*}{0.0393} & F1 & 0.00436 \\
\hline & & F2 & 0.02620 \\
\hline & & F3 & 0.00872 \\
\hline \multirow{3}{*}{$\begin{array}{l}\text { Surface } \\
\text { decoration( } \\
\text { B4) }\end{array}$} & \multirow{3}{*}{0.0923} & G1 & 0.03130 \\
\hline & & G2 & 0.00994 \\
\hline & & G3 & 0.05105 \\
\hline \multirow{2}{*}{$\begin{array}{l}\text { Function } \\
\text { (B5) }\end{array}$} & \multirow{2}{*}{0.0952} & H1 & 0.01586 \\
\hline & & H2 & 0.07933 \\
\hline \multirow{2}{*}{$\begin{array}{l}\text { Structure } \\
\text { (B6) }\end{array}$} & \multirow{2}{*}{0.2554} & I1 & 0.05108 \\
\hline & & I2 & 0.20432 \\
\hline \multirow{8}{*}{$\begin{array}{l}\text { Material } \\
\text { (B7) }\end{array}$} & \multirow{8}{*}{0.1191} & J1 & 0.03110 \\
\hline & & $\mathrm{J} 2$ & 0.01311 \\
\hline & & $\mathbf{J 3}$ & 0.01311 \\
\hline & & $\mathrm{J} 4$ & 0.01311 \\
\hline & & J5 & 0.00759 \\
\hline & & J6 & 0.03054 \\
\hline & & J7 & 0.08229 \\
\hline & & J8 & 0.00229 \\
\hline \multirow{3}{*}{$\begin{array}{l}\text { Economic } \\
\text { value }(\mathrm{B8})\end{array}$} & \multirow{3}{*}{0.0599} & K1 & 0.03753 \\
\hline & & K2 & 0.00560 \\
\hline & & K3 & 0.01675 \\
\hline
\end{tabular}


Table 4.Consistency Index (Cl) and Consistency Ratio (CR) of Matrix

\begin{tabular}{|c|c|c|}
\hline Layers & Consistency index (CI) & Consistency ratio (CR) \\
\hline A-B layer & 0.0355 & 0.0252 \\
\hline B1-C layer & 0 & 0 \\
\hline B2-C layer & 0.0470 & 0.0904 \\
\hline B3-C layer & 0 & 0 \\
\hline B4-C layer & -0.0930 & -0.1788 \\
\hline B5-C layer & 0 & 0 \\
\hline B6-C layer & 0 & 0 \\
\hline B7-C layer & 0.0302 & 0.0214 \\
\hline B8-C layer & 0.0429 & 0.0825 \\
\hline
\end{tabular}

Table 5. Evaluation Data of Upholstered Furniture Design Projects

\begin{tabular}{|c|c|c|c|c|c|c|c|}
\hline $\begin{array}{r}\text { Design } \\
\text { project }\end{array}$ & D1 & D2 & E1 & E2 & $\cdots$ & K2 & K3 \\
\hline $\mathbf{1}$ & 8 & 6 & 7 & 7 & $\cdots$ & 7 & 7 \\
\hline $\mathbf{2}$ & 8 & 7 & 9 & 9 & $\cdots$ & 7 & 7 \\
\hline $\mathbf{3}$ & 7 & 6 & 7 & 6 & $\cdots$ & 6 & 6 \\
\hline $\mathbf{4}$ & 8 & 9 & 8 & 7 & $\cdots$ & 8 & 7 \\
\hline $\mathbf{5}$ & 8 & 8 & 8 & 7 & $\cdots$ & 8 & 7 \\
\hline $\boldsymbol{\cdots}$ & $\cdots$ & $\cdots$ & $\cdots$ & $\cdots$ & $\cdots$ & $\cdots$ & $\cdots$ \\
\hline $\mathbf{9 0}$ & 8 & 8 & 7 & 7 & $\cdots$ & 8 & 6 \\
\hline
\end{tabular}

\subsection{Evaluation Model Implementation}

Firstly, the weights of upholstered furniture design comprehensive evaluation index are determined by using AHP as shown in Table 3. Then, the training samples are inputted to BP neural network for training, the BP network structure is shown in Figure 3.

\subsubsection{BP Neural Network Parameters}

A three-tier BP neural network model with one input layer, two hidden layers and one output layer were developed. The input target of system is evaluation index of evaluation system, which is the input layer with 26 nodes, the number of evaluation index. The output target of system is evaluation result of evaluation system, which is the output layer with 1 node. The number of nodes in first hidden layer is 7, and the number of nodes in second hidden layer is 2 . Number of samples is 90 , learning rate is 0.05 , and momentum parameter is 0.01 . 


\subsubsection{BP Neural Network Model Establishment}

In command window of MATLAB 2014a, a network is established by writing and inputting a program as following:

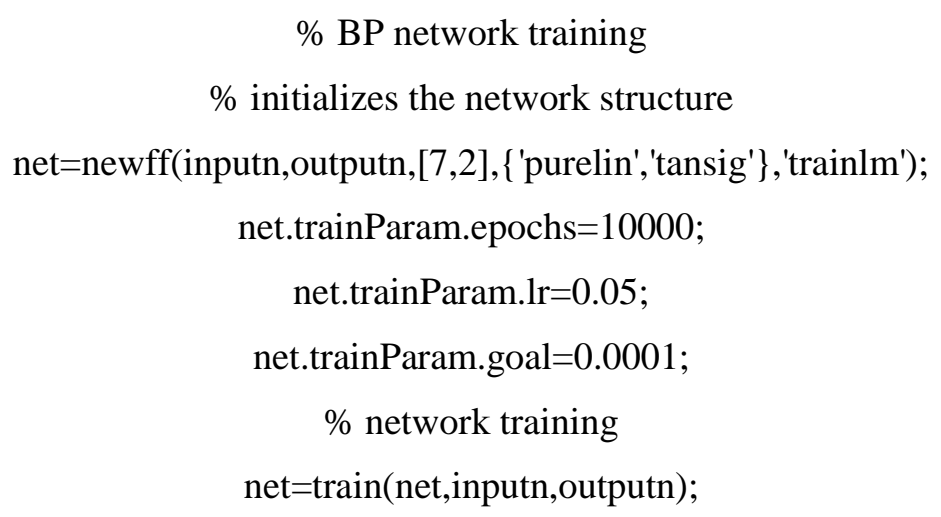

The mean square error is 0.889 before training, and the final mean square error is $9.73 \times 10-5$ after network convergence, which is shown in Figure 3. The training accuracy reaches 0.0001 at 12th training, and network is completed with network convergence, which is shown in Figure 4.

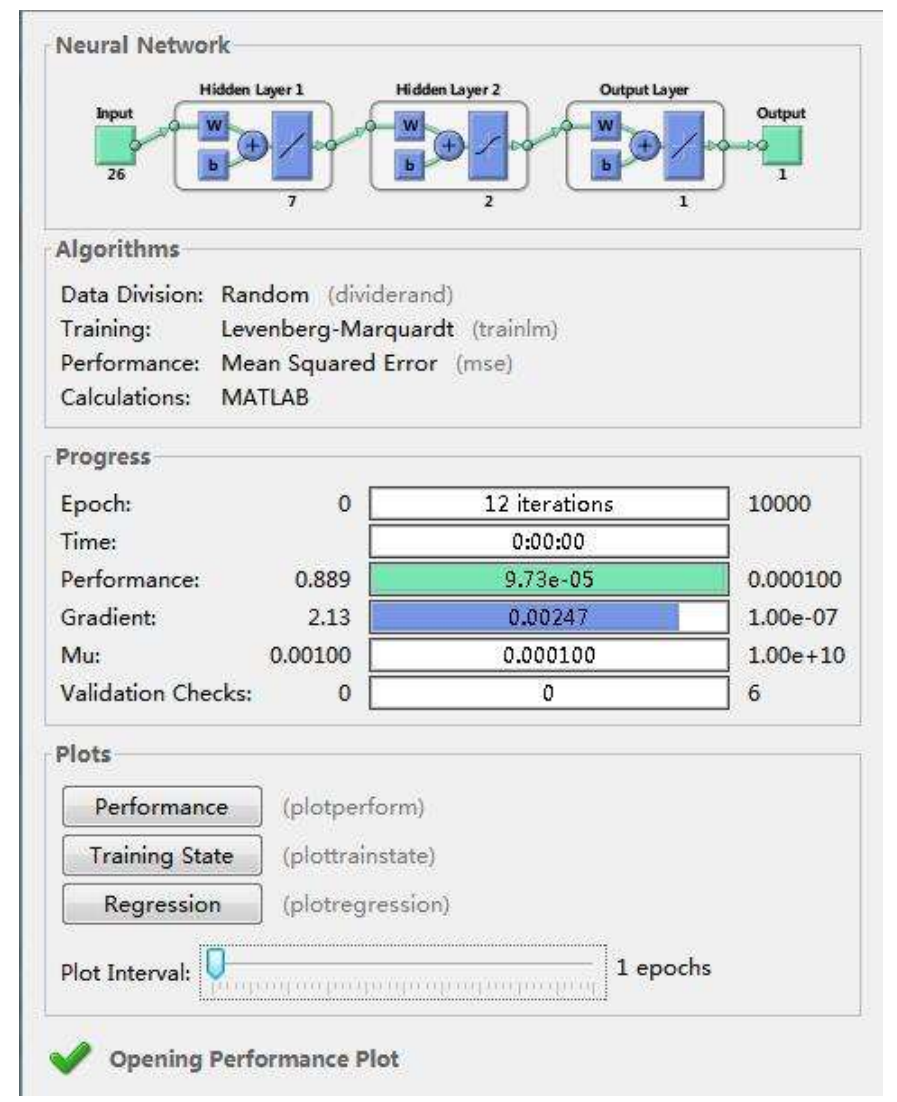

Figure 3. BP Network Structure 


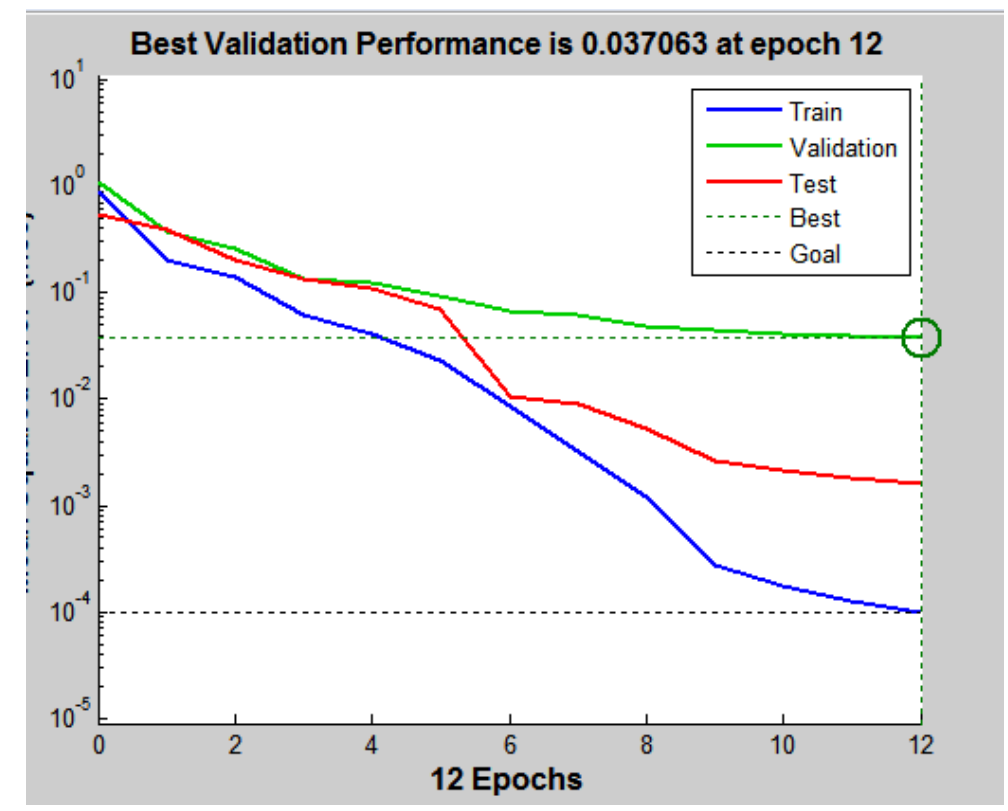

Figure 4. BP Network Training Error

\title{
4.2.3. BP Neural Network Model Prediction
}

In command window of MATLAB 2014a, a network prediction is performed by writing a program as following:

\author{
$\%$ BP network predicting \\ $\%$ predicted data normalization \\ inputn_test=mapminmax('apply',input_test,inputps); \\ $\%$ network predicting output \\ an=sim(net,inputn_test); \\ $\%$ network output anti-normalization \\ BPoutput=mapminmax('reverse',an,outputps); \\ $\%$ result analysis \\ Figure (1) \\ Plot (BPoutput,':og') \\ hold on \\ plot(output_test,'-*'); \\ legend(' predicting output ',' desired output ') \\ title(' BP network predicting output','fontsize',15) \\ ylabel(' function output ','fontsize',15) \\ xlabel(' number of times ','fontsize',15) \\ $\%$ prediction error \\ error=BPoutput-output_test
}




$$
\begin{gathered}
\mathrm{wc}=\mathrm{abs}(\text { output_test-BPoutput)./output_test } \\
\mathrm{ycwc}=\operatorname{sum}(\text { abs(output_test-BPoutput)./output_test) }
\end{gathered}
$$

Figure (2)

$$
\text { plot(error,'-*') }
$$

title('BP neural network prediction error','fontsize',12)

ylabel(' error ','fontsize',12)

xlabel(' number of times ','fontsize',12)

Figure (3)

$$
\text { plot((output_test-BPoutput)./BPoutput,'--*'); }
$$

title(' prediction error percentage of BP neural network')

$$
\text { errorsum=sum(abs(error)) }
$$

As shown in Figure 5, network are tested by 30 samples, predicting output and desired output curves overlap gradually, which indicates that network test meet the requirements, and the neural network is reasonable and effective. As shown in Figure 6, after 30 samples testing, the BP network prediction error value gradually tend to be 0 , that means the network error precision meet the requirements, the BP network prediction error percentage gradually also tend to be 0 after 30 samples testing as shown in Figure 7 . The above-mentioned figures and data indicate that network prediction result is good, neural network after prediction can be used for simulation of upholstered furniture design comprehensive evaluation.

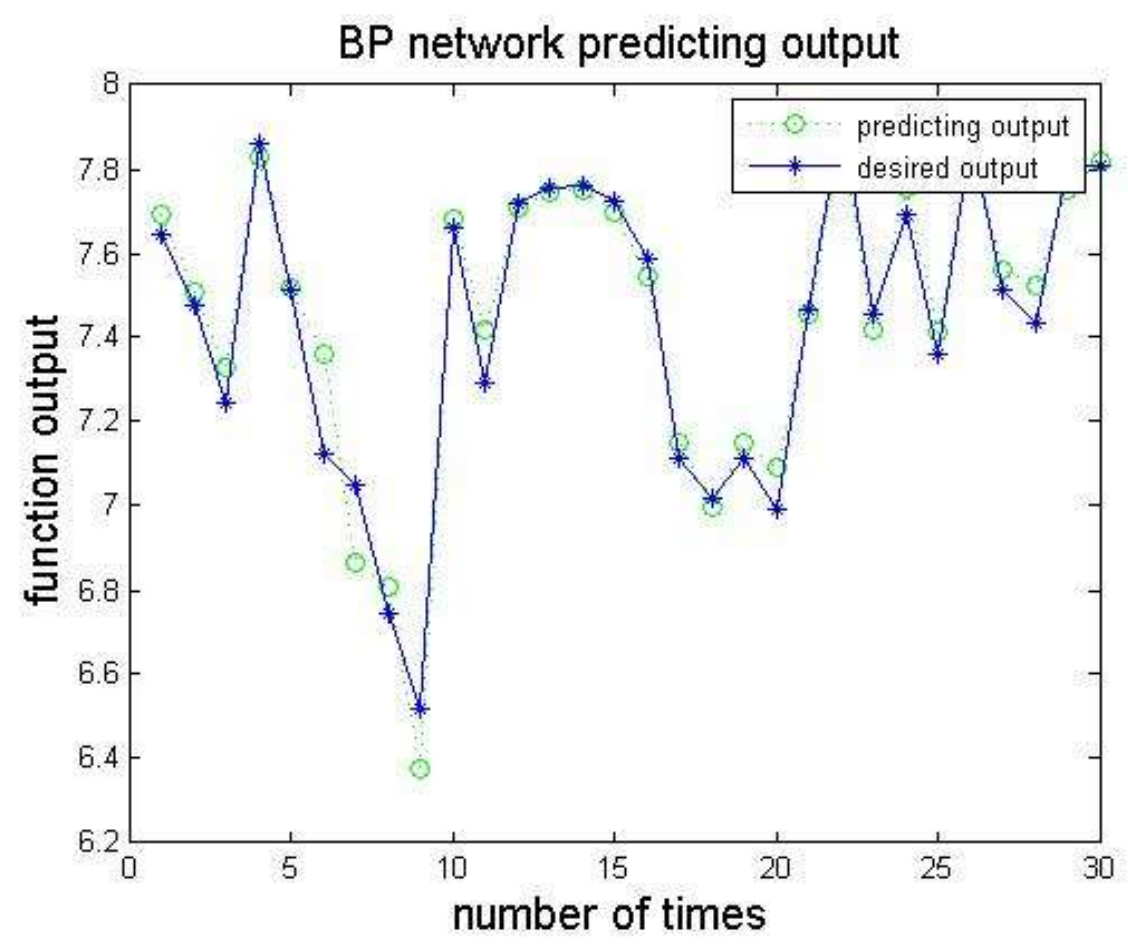

Figure 5. BP Network Predicting Output 


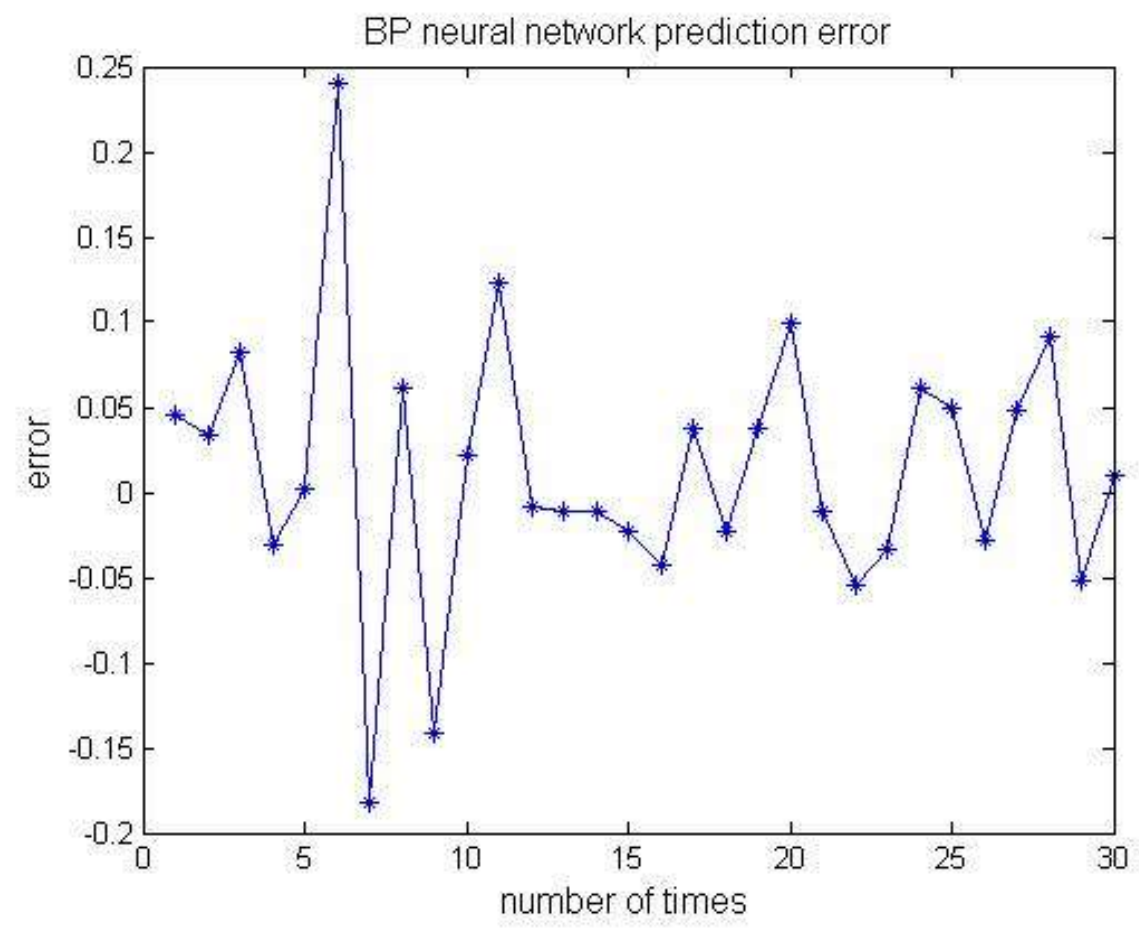

Figure 6. BP Neural Network Prediction Error

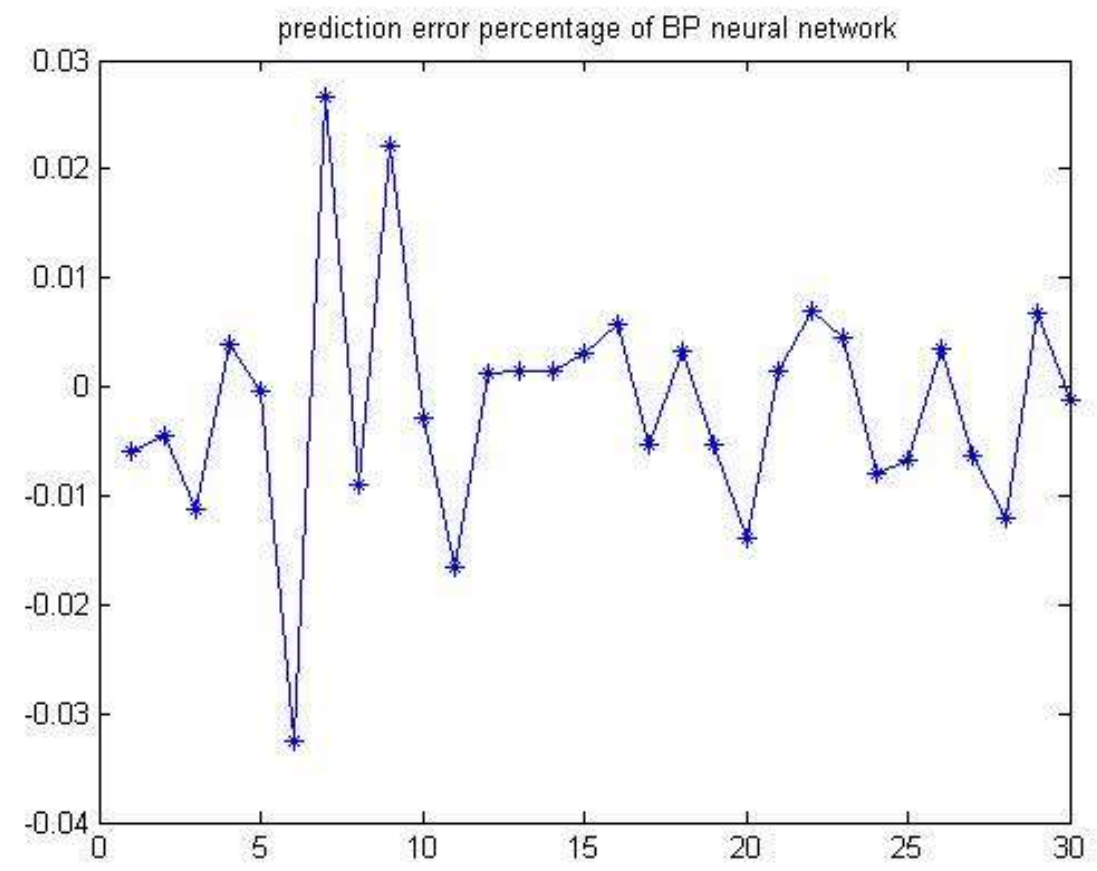

Figure 7. Prediction Error Percentage of BP Neural Network 


\section{Conclusion}

As for the issue of upholstered furniture design, a method of upholstered furniture design comprehensive evaluation based on AHP-BP neural network is proposed and performance testing is performed through simulation experiments, and the following conclusions are obtained. The use of neural network to evaluate complex upholstered furniture design quality and use of AHP to screen the importance index not only simplifies the model structure of the neural network, while improving the evaluation accuracy of upholstered furniture design quality, thereby dramatically reducing computation time of model and improving operational efficiency of upholstered furniture design quality evaluation system. Simulation results show that for evaluation of upholstered furniture design quality based on AHP-BP neural network model, the evaluation results are more scientific and accurate, with good prospects in upholstered furniture design evaluation management and decision-making.

\section{Acknowledgments}

This research is supported by the Open Research Subject of Key Laboratory (Research Base) of Industrial Design Industry Research Center (Grant No. GY-15ZD-01) and Modern Design and Culture Research Center of Sichuan Provincial Key Research Base of Philosophy and Social Sciences (Grant No. MD15E017). This research was carried out at the Sichuan Provincial Colleges and Universities Wood Industry and Furniture Engineering Key Laboratory, Sichuan Agricultural University. We are grateful to all participants for their participation and assistance in the experimental study.

\section{References}

[1] S. H. Hsu, M. C. Chuang and C. C. Chang, "A semantic differential study of designers' and users' product form perception”, International Journal of Industrial Ergonomics, vol.25, no.4, (2000), pp.375391.

[2] K. Fukushima, H. Kawata, Y. Fujiwara and H. Genno, "Human sensory perception oriented image processing in a color copy system", International Journal of Industrial Ergonomics, vol.15, no.1, (1995), pp.63-74.

[3] M. H. Yun, S. H. Han, S. W. Hong and J. Kim, "Incorporating user satisfaction into the look-and-feel of mobile phone design", Ergonomics, vol.46, no.13-14, (2003), pp. 1423-1440.

[4] S. Ishihara, K. Ishihara, M. Nagamachi and Y. Matsubara, "An automatic builder for a Kansei engineering expert system using self-organizing neural networks", International Journal of Industrial Ergonomics, vol.15, no. 1, (1995), pp.13-24.

[5] T. Jindo and K. Hirasago, "Application studies to car interior of Kansei engineering", International Journal of Industrial Ergonomics, vol.19, no.2, (1997), pp.105-114.

[6] S. M. Yang, M. Nagamachi and S. Y. Lee, "Rule-based inference model for the Kansei Engineering System”, International Journal of Industrial Ergonomics, vol.24, no.5, (1999), pp.459-471.

[7] M. Perona and N. Saccani, "Integration techniques in customer-supplier relationships: An empirical research in the Italian industry of household appliances", International Journal of Production Economics, vol.89, no.2, (2004), pp.189-205.

[8] H. H. Lai, Y. C. Lin, C. H. Yeh and C. H. Wei, "User-oriented design for the optimal combination on product design", International Journal of Production Economics, vol.100, no.2, (2006), pp.253-267.

[9] S. W. Hsiao and H. C. Tsai, "Applying a hybrid approach based on fuzzy neural network and genetic algorithm to product form design", International Journal of Industrial Ergonomics, vol.35, no.5, (2005), pp.411-428.

[10] J. Lampinen, "Cam shape optimisation by genetic algorithm", Computer-Aided Design, vol.35, no.8, (2003), pp.727-737.

[11] M. Sevaux and Y. Mineur, "A curve-fitting genetic algorithm for a styling application”, European Journal of Operational Research, vol.179, no.3, (2007), pp. 895-905. 
[12] S. W. Hsiao, F. Y. Chiu and S. H. Lu, "Product-form design model based on genetic algorithms", International Journal of Industrial Ergonomics, vol.40, no.3, (2010), pp. 237-246.

[13] T. L. Saaty, Editor, "The Analytic Hierarchy Process: Planning, Priority Setting, Resource Allocation", McGraw-Hill, New York, (1980).

[14] T. L. Saaty, "How to make a decision: the analytic hierarchy process", European Journal of Operational Research, vol.48, no.1, (1990), pp.9-26. 
International Journal of Hybrid Information Technology

Vol. 10, No.1 (2017) 